\title{
Specific T-cell Responses to CFP10, an Secreted Antigens of Mycobacterium Tuberculosis Protein, in Chinese HIV Positive Individuals
}

\author{
Bin Zhang, Wen-hui Lun, Xing-wang Li, Qi Wang, Jun Cheng and Yu Mao
}

Objective To construct prokaryotic expression vector of CFP-10 gene, and obtain recombinant protein, and the recombinant CFP-10 protein was taken as stimulus to detect specific $\mathrm{T}$ cell responses, to set up a method to faciliate to detect potential TB infection in China.

Methods CFP-10 was cloned into inducible prokaryotic expression vector pET-32a (+) and transfected into $E$. coli BL21 (DE3). After IPTG induction, the product were verified with sodium dodecyl sulfate-polyacrylamide gel electrophoresis (SDS-PAGE) and Western blot hybridization were carried out to verify the antigenicity; the recombinant CFP-10 protein was taken as stimulus to detect specific $\mathrm{T}$ cell responses in $\mathrm{HIV}(+)$ persons with or without clinical manifestation of TB diseases, and HIV (-) controls with or without TB diseases.

Results The CFP-10 recombinant protein exsited in the form of inclusion body and accounted for $94 \%$ in total bacterial protein of $E$. coli and the molecular weight is $31 \mathrm{kD}$; Western blot confirmed the recombinant proteins had high antigenicity; our in-house ELISpot-IFN- $\gamma$ assay with recombinant antigen derived from CFP-10 proteins showed significant higher frequencies in TB patients with or without HIV infection than that in the healthy controls and only HIV $(+)$ group.

Conclusions The recombinant CFP-10 genes can be expressed successfully in prokaryotic expression system of $E$. coli and recombinant proteins with high antigenicity were obtained, which will set foundation for further study on their immunogenicity and bioinformatics. Our results proved that it is indeed true that some HIV positive patient have high frequencies of TB specific T cell responses, which maybe a clue to find latent TB infection in this population.

Key words: HIV; TB; Tuberculosis antigen; CFP-10; Prokaryotic expression; ELISpot;

$\mathrm{T}$ Tuberculosis (TB) is still a major public health problem around the world, especially in China, which is one of endemic countries with the second largest tuberculosis patients population in the world. It is estimated that there are about 5 million TB patients in China. ${ }^{1}$ Bacillus of Calmette-Guérin (BCG) vaccination is widely applied in China, but vaccination with $B C G$ confers only partial protection. AIDS epidemic in China has made mycobacterium tuberculosis (tubercle bacillus) infection more complicated. Active TB disease and potential tubercle bacillus infection are the major problems in the human immunodeficiency virus (HIV) positive population. It is very difficult to make a diagnosis when an AIDS patient complicated with extra-pulmonary tuberculosis diseases. Traditional methods for diagnosis of tubercle bacillus infection such as TSTs are not sufficient for testing tubercle bacillus infection in AIDS patients.

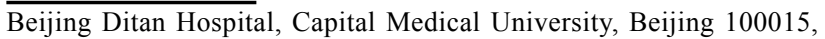
China

Correspondence: Wen-Hui Lun, E-mail:lunwenhui@163.com
Immune-based assays to detect mycobacterium tuberculosis infection is a significant advance recently. ${ }^{2}$ After tubercle bacillus infection, Th1 cellular responses are essential for efficient protection against tubercle bacillus infection in several animal models. About 5\% to $10 \%$ immunocompetent individuals could develop into active tuberculosis. ${ }^{3,4}$ In tubercle bacillus infected humans and animal models, surface or secreted antigens, especially RD-1-encoded $M$. tuberculosisspecific antigens, such as CFP-10, ESAT-6 antigens were identified as inducers of Th1 $\mathrm{CD}^{+}$cellular immune responses. ${ }^{5-7}$ As the RD-1 region is deleted in BCG strains, and can be used to differentiate between individuals with or without TB infection, regardless of BCG vaccination status. ${ }^{8-9}$ T-cell interferon gamma (IFN- $\gamma$ ) release assays (IGRAs), in which IFN- $\gamma$ production by sensitized T-cells in response to stimulation by those specific $M$. tuberculosis antigens were measured and are now licensed for the diagnosis of LTBI, ${ }^{10}$ and the sensitivity of the assay was almost 
not impaired by HIV coinfection, ${ }^{11}$ even in the advanced AIDS patients with low $\mathrm{CD} 4^{+} \mathrm{T}$ cell counts. ${ }^{12}$ However, there is insufficient evidence on IGRA performance in children and immune-compromised persons, which remain some controversial issues. ${ }^{13}$

Here, we used the recombinant CFP-10 protein as stimulus to detect specific $\mathrm{T}$ cell interferon-gamma released assays in HIV $(+)$ patients with or without clinical manifestation of tuberculosis diseases. The aim of this study is to evaluate CFP-10 specific T cell immune responses in HIV $(+)$ Chinese patients, which will provide basic data to set up CFP-10/ESAT-6 based interferon-gamma released assays to detect potential TB infection in HIV $(+)$ Chinese population.

\section{PATIENTS AND METHODS}

\section{Patients}

This study was carried out in the STD/AIDS Center of Beijing Ditan Hospital, Capital Medical University. The study protocol was approved by the institutional review board of Beijing Ditan Hospital. Patients with consecutive HIV positive were recruited according to the following inclusion criterias: $>18$ years old and nonpregnant adults. Total of 88 HIV (+) patients were divided into two groups, HIV (+) group included 73 patients whose tubercle bacillus infection situation was not clear, HIV (+)/ TB group included 15 patients who were clinically and microbiologically diagnosed as active TB infection when they were recruited.

For comparison, HIV negative controls were recruited in the same time. We recruited 7 HIV (-) TB patients from Beijing Chest Hospital, who were all $>18$ years old and microbiologically confirmed as TB patients. Also, 14 HIV negative healthy individuals aged $>18$ years were enrolled, who all had no history of TB exposure or infection.

All participants in this study had prior BCG vaccination when they were in their childhood.

\section{Recombinant of CFP-10 protein}

The primers were designed based on the lhp gene encoding the mature chain of the CFP-10 protein as recorded in GenBank (Gene accession AAC83445) and synthesized by the Shanghai Biotechnology Co. China. The primer sequences were as follows:5'CCGGATCCATGGCAGAGATGAAGAC-3' (sense), and 5'-CCAAGCTTTCAGAAGCCCATTTGC-3' (anti-sense). The underlined letters denoted the sites targeted by the restriction enzymes Bam H I /Hind III.

PCR products of the correct size were eluted from the agarose gel by a PCR purification kit (Bio Dev, China) following the supplier's protocol. The purified PCR product and pGEM-T vector (preserved by Promega, USA) were ligated by T4 DNA ligase
(Promega, USA) at $16^{\circ} \mathrm{C}$, overnight. E. coli DH5 $\alpha$ (preserved by our laboratory) was transformed with the resulting vector through heat shock. The resulting white colonies were confirmed as containing the inserted sequence by colony PCR and restrictionenzyme analysis. The Bam H I / Hind III (Promega, USA) fragment (300 bp) derived from the constructed pGEM-T-CFP10 was purified through Wizard DNA Clean-Up System (Promega). The fragment was then ligated to the same restriction sites of pET-32a vector (Institute of Infections Disease, Beijing Ditan Hospital, Capital Medical University) to obtain pET-32a-CFP10 by standard manipulation. The recombinant plasmid was transformed into $E$. coli BL21 competent cells through heat shock method. The recombinants were confirmed by restriction enzyme digestion, agarose gel electrophoresis, PCR and sequenced by the Aoke Biotechnology Co. (Beijing, China).

A correct single colony containing the expression vector was inoculated into $10 \mathrm{ml}$ LB-broth containing ampicillin $(100 \mu \mathrm{g} / \mathrm{ml})$, and the mixture was shaken at $37^{\circ} \mathrm{C}$ overnight. After further inoculation with $500 \mathrm{ml}$ LB-broth, $5 \mathrm{ml}$ culture product was cultured at $37^{\circ} \mathrm{C}$ with vigorous shaking until $A_{600}$ reached 0.6-0.8 and cells were harvested by centrifugation. The pellets were subjected to three rapid freeze-thaw cycles at $-80^{\circ} \mathrm{C}$. After sonication and centrifugation $(15,000$ $\mathrm{rpm}, 25 \mathrm{~min})$, the supernatants and pellets were resuspended in $100 \mu 11 \mathrm{~mol} / \mathrm{L}$ Tris- $\mathrm{HCl}$ (pH 6.8), 100 $\mu 12 \times$ SDS buffer solution and $5 \mu 1 \beta$-mercaptoethanol, then analyzed by $12 \%$ SDS-PAGE (sodium dodecyl sulfate polyacrylamide gel electropheresis). After electrophoresis, the gels were stained with Coomassie Blue and destained, and then protein expression yield was measured by screening light density and comparing results with molecular weight standard. The transference distance of protein was taken to calculate the related transference frequency and molecular weight (MW).

According to the optimization condition, induced cells were harvested by centrifugation at $12,000 \mathrm{rpm}$ for 10 minutes and resuspended in sonication buffer containing $0.5 \mathrm{~mol} / \mathrm{L} \mathrm{NaCl}, 20 \mathrm{mmol} / \mathrm{L} \mathrm{PB}$, then sonicated by an ultrasonic disrupter. The lysate was centrifuged at $15,000 \mathrm{rpm}$ for 20 minutes at $4{ }^{\circ} \mathrm{C}$. After filteration of supernatant, the fusion protein was purified by Amersham Bioscinces purifier (type: AKTA purifier). Recombinant protein was eluted with inidazole $(500 \mathrm{mmol} / \mathrm{L}$ glyoxalin, $0.5 \mathrm{~mol} / \mathrm{L} \mathrm{NaCl}, 20$ $\mathrm{mmol} / \mathrm{L} \mathrm{PB}$ ). After desalted and changed buffer by a routine centrifugal filter device $(10 \mathrm{kD})$, the fusion protein was analyzed by SDS-PAGE.

The fusion protein was probed with primary antibodies, the mouse-anti-His, which was diluted in blocking solution $(1: 250$, Santa Cruz), and a 
peroxidase labeled goat-anti-mouse IgG was taken as the secondary antibody $(1: 2000)$. Then the fusion protein was also probed with the serum of TB patients, diluted by PBS in blocking solution $(1: 200)$, and a peroxidase labeled goat-anti-human IgG was taken as the secondary antibody $(1: 2000)$. Then both of the specific bindings were detected by West Pico Chemiluminescent Substrate (Pierce).

\section{ELISpot-IFN- $\gamma$ assay}

Peripheral blood mononuclear cells (PBMCs) were separated from heparinized venous blood by FicollPaque centrifugation. A commercially available IFN- $\gamma$ ELISpot kit (Diaclone) containing a precoated 96-well plate was applied (Millipore). The ELISpot IFN- $\gamma$ assay was carried out with freshly prepared PBMC. The anti-human IFN- $\gamma$ capture Mab was taken to coat 96-well polyvinylidene difluoride plates, and incubated overnight at $4{ }^{\circ} \mathrm{C}$. CFP10 antigen was added to two wells (duplicate), with the concentrations of $50 \mu \mathrm{g} / \mathrm{ml}$. PBMC were cultured in complete RPMI 1640 medium which was supplemented with fetal calf serum $(10 \%$, Gibco, USA). Negative controls consisted of cells cultured with medium alone and positive controls consisted of cells cultured with Con A. PBMC $\left(2 \times 10^{5}\right.$ per well corresponding to the number of cells inducing optimal responses to antigens) were incubated for $18-20$ hours at $37^{\circ} \mathrm{C}$ in an atmosphere containing $5 \%$ $\mathrm{CO}_{2}$.

The plates were washed and a biotinylated antiIFN- $\gamma$ Mab (Diaclone) was added, followed by streptavidin-alkaline phosphatase conjugate and chromogen substrates (5-bromo-4-chloro-3-indolyl phosphate toluidine and nitroblue tetrazolium) as the instruction indicated. Plates were dried and the colored spots were counted through an automated ELISpot reader (CTL-spot, Germany). The numbers of specific $\mathrm{T}$ cells expressed as pot-forming cells per million PBMC (SFC $/ 10^{6} \mathrm{PBMC}$ ) were calculated after subtracting the mean values and obtained without antigen for each sample. According to other Researchers' experience, responses were considered positive if the test well contained at least $50 \mathrm{SFC} / 10^{6} \mathrm{PBMC}$ of IFN- $\gamma$ more than negative control wells. ${ }^{14}$

\section{Statistical analysis}

$\chi^{2}$ were applied to compare proportions and medians of the four groups as appropriate. Assay responses were evaluated as continuous (quantitative) variables and as categorical variables (positive or negative responses) according to the thresholds predefined above. Associations between patient characteristics and positive ELISpot were examined through univariate analysis and multiple logistic regression models. Data were analyzed with software SPSS 11.5.

\section{RESULTS}

\section{Patients' characteristics}

In HIV (+) group, 73 subjects (57 men and 16 women) were HIV infected patients without clinical manifestation of active TB, the mean age was 32 years old (range 22-84 years old), 3 patients of whom had a prior history of tuberculosis disease with optimal treatment, and didn't have clinical symptom for more than 5 years. The mean count of $\mathrm{CD}^{+} \mathrm{T}$ lymphocyte was 272 cells/ $\mu 1$.

In HIV $(+) / \mathrm{TB}$ group, 14 subjects (13 men and 2 women) were HIV positive patients with clinical active TB infection, with the median age as 39 years old (range 30-49 years old). The localization of tuberculosis disease was pulmonary $(\mathrm{n}=13)$, extrapulmonary $(n=1)$ or both $(n=1)$. The mean count of CD4 ${ }^{+} \mathrm{T}$ lymphocyte was 179 cells/ $\mu \mathrm{l}$.

In TB group, 7 subjects ( 6 men and 1 woman) were with active tuberculosis disease and HIV negative, with the mean age as 58 years old (range 36-70 years old).

In healthy control group, 14 healthy volunteers $(8$ men and 6 women) were enrolled, with the median age as 32 years old (range 18-49 years old). None in this group had a history of TB infection (Table 1).

\section{The recombination of CFP10 protein}

Total DNA was isolated from the whole genome of HR37v and PCR products of lhp gene resulted a 300 bp fragment. After being cut by Bam H I/Hind III, pET32a-CFP10 recombinant plasmid revealed two bands of approximately 5397 bp and 300 bp on 1\% agarose gel (Figure 1). CFP10 sequencing confirmed that lhp gene cloned was exactly matched with the sequence in GenBank.

The recombinant protein was yielded with optimizing expression conditions. ${ }^{15}$ 12\% SDS-PAGE showed that CFP10 protein had an expected distinct protein band of $31 \mathrm{kD}$, which was absent in uninduced cells. The levels of recombinant protein measured by screening light-density account for $94 \%$ of total bacterial protein. The bacterial solution was sonicated and centrifuged at $12,000 \mathrm{rpm}$ and the supernatant was compared with the original mixture through SDSPAGE. The results showed that the induced fusion protein was concentrated primarily in the supernatant, which implied that it is a soluble protein.

CFP10 protein was identified by SDS-PAGE and then confirmed by Western blot with the antibody mouse-anti-His, but the control group was negtive (Figure 2).

The recombinant CFP10 protein could also be detected by the serum of TB patient as the first antibody, but negative by the serum from healthy control who had no history of TB infection (Figure 3). 
The recombinant protein was purified and then eluted by Ni-NTA containing column. After desalting and changing the buffer, the protein was analyzed by SDS-PAGE. The concentration of recombinant protein CFP10 was $3.5 \mathrm{~g} / \mathrm{L}$ which was measured by BAC method.

\section{Results of ELISpot assays}

ELISpot IFN- $\gamma$ assay was taken to quantify CFP10specific $\mathrm{T}$ cells in both HIV positive patients and HIV negative controls, all subjects included 73 patients in HIV (+) group, 15 patients in HIV (+)/TB group, 7 HIV (-) TB patients in TB group and 14 patients in healthy controls.

In all subjects included in the analysis, the positive control stimuli produced measurable responses in ELISpot assay. Then the spot-forming units (SFU) in paired wells in $10^{6} \mathrm{PBMC}$ and the proportions of patients with assay responses categorised as positive according to the predefined thresholds were calculated (Figure 4, typical colored spots counted by automated ELISpot reader).

The magnitude of responses to CFP-10 stimuli were significantly lower in healthy controls and HIV $(+)$ group compared with TB group and HIV $(+) /$ TB group. In healthy controls, the median positive frequency is $26 \mathrm{SFC} / 10^{6} \mathrm{PBMC}$ (interquartile ranging from 10 to 43). According to the presumed criteria, there was one

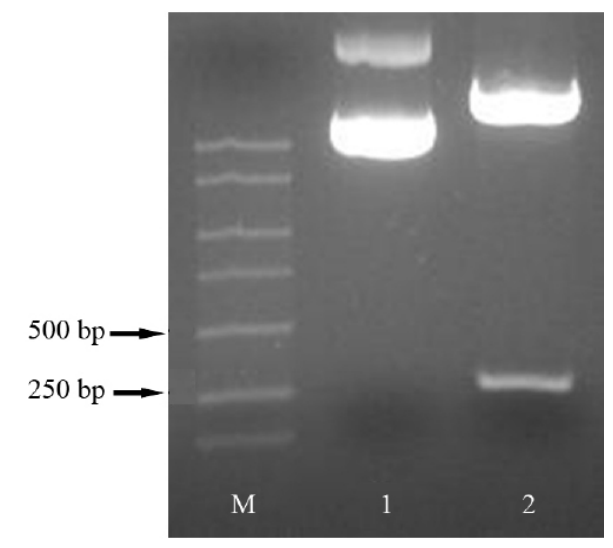

Figure 1. Identification of pET32a (+)-CFP10 cut by restriction enzyme.

Notes: Line 1, The plasmid of pET32a (+)-CFP10; Line 2, The result of double cut by Bam $\mathrm{H}$ I /Hind III; M, Marker.

After being cut by Bam H I /Hind III, pET32a-CFP10 recombinant plasmid revealed $300 \mathrm{bp}$ (CFP10) on 1\% agarose gel. positive sample in healthy controls, with the positive rate as $14.2 \%(2 / 14)$.

For HIV ( + ) group, the median positive frequency is $17 \mathrm{SFC} / 10^{6} \mathrm{PBMC}$ (interquartile ranging from 5 to 51 ), with the positive rate as $26.0 \%(19 / 73)$.

All subjects in TB group showed very high frequency of CFP-10 specific T-cell response, with the median frequency as $566 \mathrm{SFC} / 10^{6} \mathrm{PBMC}$ (interquartile ranging from 468 to 700 ) and the positive rate as $100 \%$ (7/7).

The subjects in HIV (+)/TB group also displayed high magnitude of CFP-10 specific T-cell responses, with the median frequency as $168 \mathrm{SFC} / 10^{6} \mathrm{PBMC}$ (ranging from $91-345$ ), and the positive rate as $100 \%$ $(15 / 15)$.

The frequencies of CFP10-specific $\mathrm{T}$ cell responses was significantly higher in TB group and HIV $(+) / T B$ group. The median numbers of spot forming unit were significantly higher in either HIV (+)/TB group and TB group compared with that in HIV $(+)$ group $(P<$ $0.05)$ or healthy group $(P<0.05)$. However, the spot forming unit was significantly high in several patients in HIV (+) group, which may indicate potential TB infection in those HIV $(+)$ patients.

There was no relationship between CFP-10 specific $\mathrm{T}$ cell responses and age or sex in all 4 groups, also no relationship between CFP-10 specific $T$ cell response and $\mathrm{CD}^{+} \mathrm{T}$ cell counts in both of HIV (+)/TB group

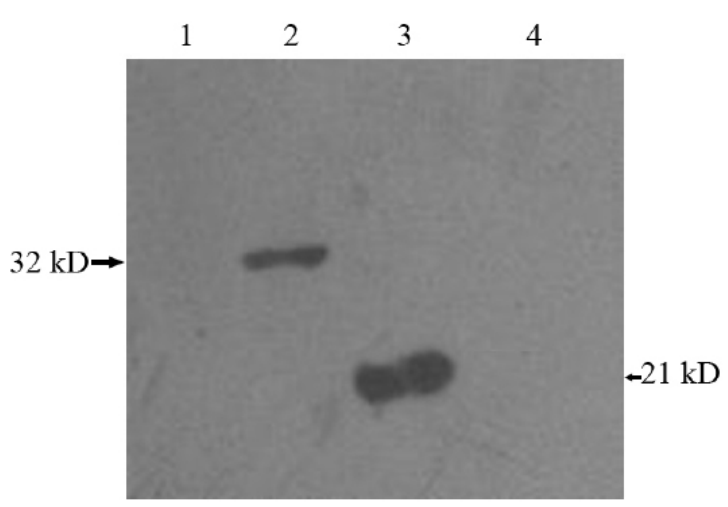

Figure 2. The Western blotting result of pET32a (+)-CFP10, pET32a (+) as control.

Notes: Line 1-2, cell lysates of E. coli strain BL21 (DE3) containing pET32a (+)-CFP10 not induction and induction by IPTG; Line 3-4: pET32a $(+)$ expressed in BL21 under induction and not induction. pET32aCFP10 protein was confirmed by Western blot with the antibody mouseanti-His, but control group was negative.

Table 1. Characteristics of patients and controls

\begin{tabular}{|c|c|c|c|c|c|c|}
\hline \multirow{2}{*}{ Groups } & \multirow{2}{*}{ Number } & \multirow{2}{*}{$\begin{array}{c}\text { Gender } \\
(\mathrm{M} / \mathrm{F})\end{array}$} & \multirow{2}{*}{$\begin{array}{c}\text { Median age } \\
\text { (years) }\end{array}$} & \multicolumn{3}{|c|}{ CD4 counts distribution } \\
\hline & & & & $\leqslant 200$ & $200-350$ & $\geqslant 350$ \\
\hline Healthy controls & 9 & $6 / 3$ & 32 & - & - & - \\
\hline TB & 7 & $6 / 1$ & 40 & - & - & - \\
\hline $\mathrm{HIV}(+) / \mathrm{TB}$ & 14 & $12 / 2$ & 58 & 15 & 17 & 14 \\
\hline $\mathrm{HIV}(+)$ & 46 & $34 / 12$ & 32 & 8 & 4 & 1 \\
\hline
\end{tabular}




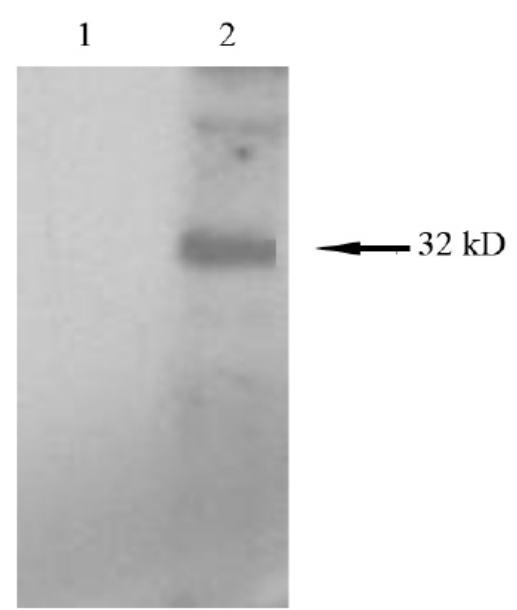

Figure 3. The Western blotting result of CFP10, with the health adult as control.

Notes: Line 1, the serum of health adult as the first antibody; Line 2, the serum of TB patient as the first antibody. The recombinant CFP10 protein could also be detected by the serum of TB patient as the first antibody, but negative by the serum from healthy control who had no history of TB infection.

and HIV $(+)$ group (Table 2 ). $\mathrm{CD}^{+} \mathrm{T}$ cell counts were relatively lower in most patients in HIV $(+)$ TB group, but very strong CFP-10 speific INF- $\gamma$ positive $\mathrm{T}$ cell responses still could be detected (Figure 5).

\section{DISCUSSION}

CFP-10 is one of the proteins encoded by RD1 genomic segment of $M$. tuberculosis, which is absent in all BCG strains and most of the other environmental mycobacteria. ${ }^{6,7}$ Enzyme-linked immuno-spot (ELISpot) assays which may detect interferongamma (IFN- $\gamma$ ) release in response to these antigens could differentiate $M$. tuberculosis infection from the individuals sensitised by BCG vaccination. ${ }^{16}$ The sensitivity of this assay was almost not impaired by HIV co-infection. Even in patients with advanced AIDS, the proportion of positive ELISpot responses was independent of $\mathrm{CD} 4^{+}$cell counts. ${ }^{12}$

Endemic $M$. tuberculosis infection and BCG vaccination are common in China. Although BCG vaccination is widely used in China, it confers only partial protection. And HIV/AIDS makes TB endemic more complicated in China. HIV positive population naturely represent part of high-risk populations to be infected by TB, but the difficulty of diagnosis is due to the sensitivity and specificity of routine tests such as TST, which are lower due to the immunodeficiency and cross-reaction of PPD with vaccine BCG strains. So a new strategy for specific diagnosis is necessary in China.

In this study, we use the recombinant CFP-10 protein as stimulus to detect $\mathrm{T}$ cell response to TB infection in
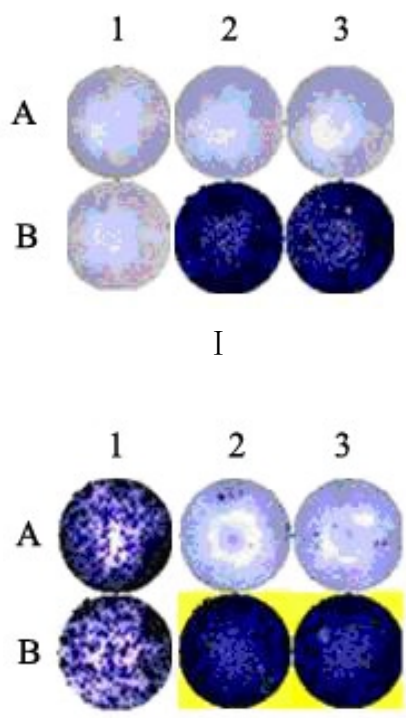

II

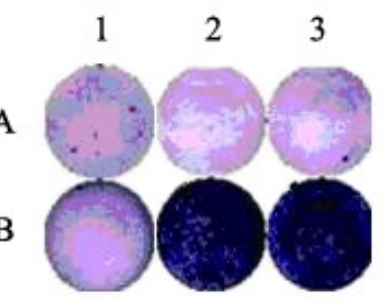

III

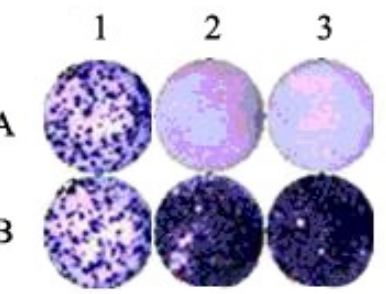

IV

Figure 4. Colored spots of typical samples counted by an automated ELISpot reader.

Notes: I , Healthy controls; II, TB patients (TB); III, HIV positive without clinical TB diseases HIV (+); IV, HIV infected patients with TB diseases

A1, B1 consisted of cells cultured with fusion protein CFP10 $(25 \mu \mathrm{g} / \mathrm{ml})$; A2, A3 negative controls consisted of cells cultured with medium alone; B2, B3 positive controls consisted of cells cultured with Con A (25 $\mu \mathrm{g}$ / $\mathrm{ml})$.

I : Cells in A1, A2, A3, B1, B2, B3 were 1, 0, 0, 1, 323, 299, respectively; II : Cells in A1, A2, A3, B1, B2, B3 were 224, 6, 5, 258, 738, 782, respectively;

III: Cells in A1, A2, A3 ,B1, B2, B3 were 3, 0, 1, 2, 207, 174, respectively; IV: Cells in A1, A2, A3, B1, B2, B3 were 165, 0, 1, 162, 275, 283, respectively; 


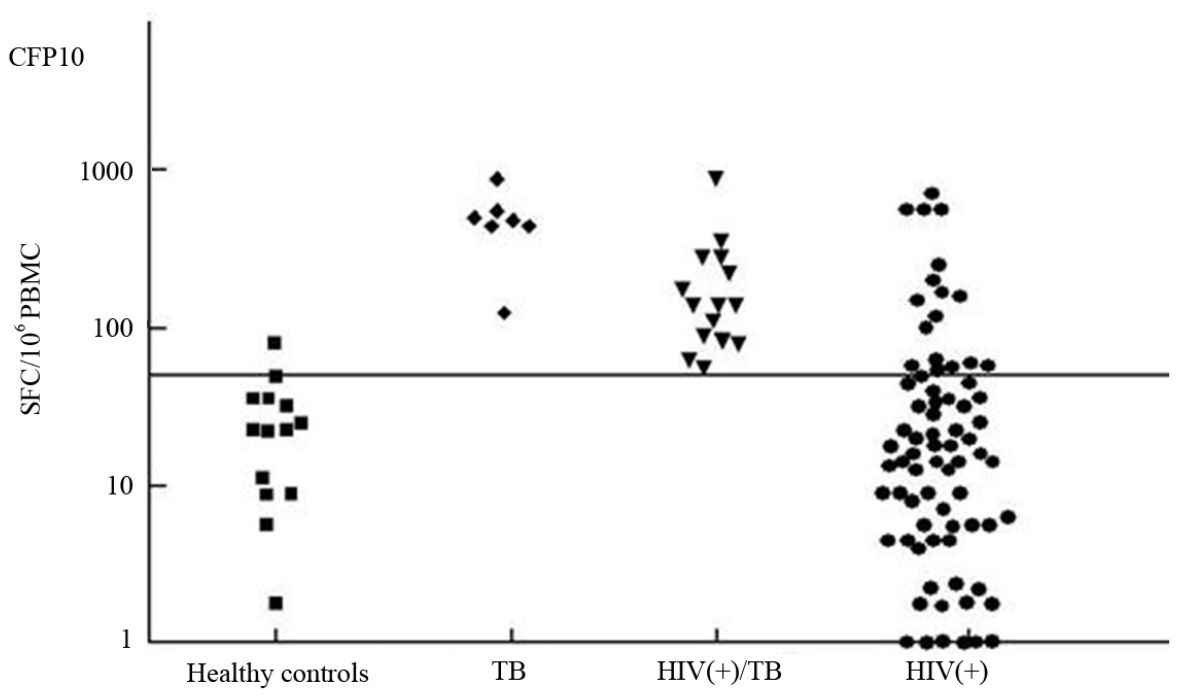

Figure 5. The result of the IFN- $\gamma$ responses detected in ELISpot assays.

Notes: Numbers of antigen-specific T cells in the 4 groups: Healthy controls , TB patients (TB) and HIV infected patients with TB diseases [HIV (+)/TB], and HIV positive without clinical TB diseases [HIV $(+)]$, measured by ELISpot IFN- $\gamma$ assay after stimulation with CFP-10. A logarithmic scale is used and horizontal bars indicate positive values.

HIV positive population and HIV negative controls. We collected basic data for interferon-gamma (IFN- $\gamma$ ) releasing based assay. The results showed significant higher frequencies in both TB patients with or without HIV infection, all samples from both TB with and without HIV infection group were detected as positive. And it showed no relationship between frequencies of CFP-10 specific $\mathrm{T}$ cell responses and $\mathrm{CD} 4^{+} \mathrm{T}$ cell counts of HIV infected patients. It is proved that this kind of assay can be used to differentiate individuals with TB infection, regardless of BCG vaccination or HIV infection status. ${ }^{17}$

For healthy controls, they were BCG-vaccinated individuals with no history of TB exposure or infection. Most of them showed negative results, however, $\mathrm{T}$ cell responses in 1 healthy case showed relatively high frequency of antigen specific $\mathrm{T}$ cell responses (94 SFU $/ 10^{6}$ cells), with the possible explanation as the exposure to $M$. tuberculosis, this case with high frequency of CFP-10 specific T cell responses was potentially corresponding to latent TB infection despite no reported TB contaction. China is one of the TB endemic countries, it is possible to find latent TB infection in those persons who have no symptoms or reported TB exposure.

In HIV (+) group, all the participants did not have any symptoms of TB diseases, about $28.3 \%(13 / 46)$
HIV patients had high frequency of CFP-10 specific $\mathrm{T}$ cell responses. So it is possible that there may be some HIV infected patients with latent TB infection. Interestingly, the three patients with history of cured tuberculosis disease longer than 5 years showed low frequency of $\mathrm{T}$ cell responses. It is indicated that TB infection history with successful treatment would not affect the results of IFN- $\gamma$ based ELISpot assay in HIV positive persons. ${ }^{18}$

China is one of the TB highly endemic countries. HIV positive population is especially vulnerable to TB infection. It is very important to evaluate $\mathrm{T}$ cell responses to each RD-1 encoded TB antigen in HIV population. We had discribed the EAST-6 specific T cell resoponses in HIV infected individuals before. ${ }^{19}$ This study showed the primary results of IFN- $\gamma$ based ELISpot assay with CFP-10 as stimulus, with the results as high frequencies of TB specific $\mathrm{T}$ cell responses in some HIV positive patients. However, in the future, the assay with both EAST-6 and CFP-10 as stimulus is necessary to evaluate if those with high frequency of $\mathrm{T}$ cell responses are latent TB infection in HIV positive population.

Table 2. Quantitative IFN- $\gamma$ responses to CFP-10 in HIV positive patients

\begin{tabular}{ccccc}
\hline Groups & & $\mathrm{CD}^{+} \leqslant 200$ & $\mathrm{CD}^{+} 200-350$ & $\mathrm{CD}^{+}>350$ \\
\hline \multirow{2}{*}{$\mathrm{HIV}(+)$} & $\mathrm{N}$ & 15 & 17 & 14 \\
& $\mathrm{SFU} / 10^{6}$ & $16(2-660)$ & $40(5-637)$ & $18(1-168)$ \\
$\mathrm{HIV}(+) / \mathrm{TB}$ & $\mathrm{N}$ & 8 & 4 & 1 \\
& $\mathrm{SFU} / 10^{6}$ & $118(61-1065)$ & $295(163-345)$ & 190 \\
\hline
\end{tabular}




\section{REFERENCES}

1. Dye C, Scheele S, Dolin P, Pathania V, Raviglione MC. Consensus tatement global burden of tuberculosis: estimated incidence, prevalence, and mortality by country. WHO global Surveillance and Monitoring Project. JAMA 1999;282:677-686.

2. Pai M, Riley LW, Colford JM Jr. Interferon-gamma assays in the immunodiagnosis of tuberculosis: a systematic review. Lancet Infect Dis 2004:4:761-776.

3. Chan J, Flynn J. The immunological aspects of latency in tuberculosis. Clin Immunol 2004;110:2-12.

4. Flynn JL, Chan J. Immunology of tuberculosis. Annu Rev Immunol 2001;19:93-129.

5. Andersen P, Andersen AB, Sorensen AL, Nagai S. Recall of longlived immunity to Mycobacterium tuberculosis infection in mice. J Immunol 1995;154:3359-3372.

6. Mustafa AS, Amoudy HA, Wiker HG, Abal AT, Ravn P, Oftung $\mathrm{F}$, et al. Comparison of antigen specific T-cell responses of tuberculosis patients using complex or single antigens of Mycobacterium tuberculosis. Scand J Immunol 1998;48:535-543.

7. Masungi C, Temmerman S, Van Vooren JP, Drowart A, Pethe K, Menozzi FD, et al. Differential T and $\mathrm{B}$ cell responses against Mycobacterium tuberculosis heparin-binding hemagglutinin adhesin in infected healthy individuals and patients with tuberculosis. J Infect Dis 2002;185:513-520.

8. Lewis KN, Liao R, Guinn KM, Hickey MJ, Smith S, Behr MA, et al. Deletion of RD1 from Mycobacterium tuberculosis mimics Bacilli Calmette-Guerin attenuation. J Infect Dis 2003; 187:117-123.

9. Gordon SV, Brosch R, Billault A, Garnier T, Eiglmeier K, Cole ST. Identification of variable regions in the genomes of tubercle bacilli using bacterial artificial chromosome arrays. Mol Microbiol 1999;32:643-656.

10. Pai M, Riley LW, Colford JM Jr. Interferon-gamma assays in the immunodiagnosis of tuberculosis: a systematic review. Lancet Infect Dis 2004; 4:761-776.

11. Rangaka MX, Wilkinson KA, Seldon R, Van Cutsem G, Meintjes GA, Morroni C. Effect of HIV-1 infection on T-Cell-based and skin test detection of tuberculosis infection. Am J Respir Crit Care Med 2007; 175:514-520.

12. Lawn S, Bangani1 N, Vogt M, Bekker LG, Badri M, Ntobongwana $\mathrm{M}$, et al. Utility of interferon- $\gamma$ ELISPOT assay responses in highly tuberculosis-exposed patients with advanced HIV infection in South Africa. BMC Infectious Diseases 2007;7:99.

13. Menzies D, Pai M, Comstock G. Meta-analysis: new tests for the diagnosis of latent tuberculosis infection: areas of uncertainty and recommendations for research. Ann Intern Med 2007; 146:340-354

14. Jeffries DJ, Hill PC, Fox A, Lugos M, Jackson-Sillah DJ, Adegbola RA, et al. Identifying ELISPOT and skin test cut-offs for diagnosis of Mycobacterium tuberculosis infection in The Gambia. Int J Tuberc Lung Dis 2006;10:192-198.

15. Zhang B, Lun WH, Chen J, Zhao LF, Mao Y, Li XW, et al. Prokaryotic expression and bioinformatics comparison of tuberculosis antigen ESAT-6 and CFP-10 recombinant protein and rCFP10-ESAT6 fusion protein. Zhonghua Shi Yan He Lin Chuang Gan Ran Bing Za Zhi (Chin) 2008;2:33-43.

16. Lalvani A, Pathan AA, Durkan H, Wilkinson KA, Whelan A, Deeks JJ, et al. Enhanced contact tracing and spatial tracking of Mycobacterium tuberculosis infection by enumeration of antigenspecific T cells. Lancet 2001;357:2017-2021.

17. Lalvani A, Pathan AA, McShane H, Wilkinson RJ, Latif M, Conlon CP, et al. Rapid detection of Mycobacterium tuberculosis infection by enumeration of antigen-specific T cells. Am J Respir Crit Care Med 2001;163:824-828.

18. Aiken AM, Hill PC, Fox A, McAdam KP, Jackson-Sillah D, Lugos MD, et al. Reversion of the ELISPOT test after treatment in Gambian tuberculosis cases. BMC Infectious Diseases 2006;6:66.

19. Zhang B, Lun WH, Cheng J, Zhao LF, Li XW, Han N, et al. Specific T-cell responses to Mycobacterium tuberculosis protein ESAT-6 in Chinese HIV positive individuals. Zhonghua Shi Yan He Lin Chuang Bing Du Xue Za Zhi (Chin) 2008;22:124-126. 